\title{
An Advanced BEM for Thermal and Stress Analyses of Components with Thermal Barrier Coating ${ }^{1}$
}

\author{
Shan Lu and Menhong Dong \\ P. O. Box 184, Northwestern Polytechnical University \\ Xi'an, Shaanxi 710072, P. R. China
}

\begin{abstract}
An advanced boundary element method (BEM) for 2-D steady-state thermal analysis of components with thermal barrier coating (TBC) is presented in this paper. First, a scheme of evaluating the nearly singular integrals in stress analysis of a thin body was extended to the thermal analysis of components with TBC. Then the BEM formulation for thermal analysis of multi-layered structures was developed.

Next, an advanced BEM was developed for 2-D interfacial stress analysis of components with TBC. The non-linear transformation scheme in stress analysis of thinbody under traction load by BEM was extended to evaluate the nearly singular integral in BEM for thermal and centrifugal stress analysis. Then BEM formulation for multi-layer structure under thermal, centrifugal and traction loads was presented.
\end{abstract}

Several numerical examples of 2-D thermal analyses and interfacial stress analyses of components with TBC were analyzed with only 32 boundary elements. The accurate numerical results can be obtained even when the ratio of the coating thickness to element length approached $10^{-9}$. The maximum relative percentage errors of interfacial tangent tension stresses under a complex temperature field, traction and centrifugal loads were $0.08 \%, 0.13 \%$ and $0.23 \%$, respectively, The present BEM will be an efficient tool for TBC design and TBC peeling-failure analysis.

\section{Introduction}

As the demand on aircraft mobility, low cost and reliability increases, the demand on aero-engine performance and reliability increases as well. Therefore aero-engine with a large thrust and a high ratio of thrust to weigh is needed. Increasing temperatures in the front of a turbine is one of the most efficient way to increase thrust and ratio of thrust to weigh. When the temperature before a turbine is higher than $1300 \mathrm{~K}$, a blade made of single metal can not endure such a high temperature. By use of thermal barrier coating technology, the highest temperature on substance can decrease $100-170{ }^{0} C^{[1]}$. But whenever thermal barrier coating peels off, the substance behind it will over-heat and

\footnotetext{
${ }^{1}$ Acknowledgements: The first author (SL) would like to acknowledge Professor Yijun Liu at the University of Cincinnati for valuable discussions and providing reference information. This project has been supported by the Aeronautical Science Foundation of China under contract No. 99c53026, Research Foundation for Returned Oversea Scholars provided by Chinese Ministry of Education and Research Startup Fund provided by Northwestern Polytechnical University.
} 
burn out. It is important to have an efficient numerical analysis tool for designing and analyzing thermal barrier coated components to optimum the distribution of their temperature and stresses, to have the coating system a durable life. Nowadays, the most commonly used stress analysis tools of coated components were engineering simplified $\operatorname{method}^{[1]}$, finite element method ${ }^{[2,3,4]}$ and boundary element method ${ }^{[5]}$. Using the engineering simplified method ${ }^{[1]}$, the stress and strain field of substrate was first obtained without considering the existing coatings. Then stress and strain fields of coatings were approximated according to the continuity condition of the substrate and the coatings. The scheme is simple and easy to use, but its accuracy and effectiveness depend on the stiffness ratio of the coatings to the substrate. And usually, a constant stress distribution in the coating is assumed along the thickness direction. Finite element method is one of the most powerful numerical tools. But for the stress analysis of coating systems, the shell element can not be used, as the shear and the normal stresses on the interface can not be simulated by shell elements. When using the brick element to analyze stresses of the coating system, mesh with a similar size to the coating thickness has to be used, as the sizes in three dimensions of a brick element should be in a suitable range ${ }^{[2,3,4]}$. Therefore very fine elements should be used with a very large number of elements and huge demand of the storage space and CPU time for the computation. When using the conventional BEM to analyze stresses of coating system, a problem with nearly singular integrals will be encountered. Similarly to the finite element method, very fine boundary elements, with a size of the coating thickness, should be used. Therefore, the stress analysis of the coating system by conventional BEM was not commonly seen in the literatures, until the research of BEM for stress analysis of a thin body achieved a breakthrough ${ }^{[5,6]}$ recently. Nowadays, thermal stress analysis of coating systems by the BEM for thin body stress analysis was limited to problems with a constant temperature field ${ }^{[7]}$, as an effective numerical method for thermal analysis of the coating system was needed, on which less research works were found in the literature. Before, the thermal analysis of a coating system was done by the finite difference method ${ }^{[8]}$, but it is difficulty to analyze a problem with a complex shape. When doing the thermal analysis of a coating system by FEM, very fine elements, with sizes of the coating thickness, should be used, as stress analysis of the coating system by FEM, which was inefficient ${ }^{[3]}$. In order to perform the thermal stress analysis of the coating system, the scheme to evaluate the nearly singular integral in thin body stress analysis ${ }^{[6]}$ will be improved and extended to thermal stress analysis of the coating system under centrifugal load and complex temperature fields. Furthermore, a BEM for thermal analysis of the coating system is proposed. Finally, several numerical examples of thermal analysis and thermal stress analysis of the coating system are given to demonstrate the efficiency of the method presented in this paper.

\section{Boundary integral equations for thermal analysis and treatment of nearly singular integrals}

Boundary integral equations for steady state temperature field analysis of an isotropy body can be expressed as ${ }^{[9]}$ 


$$
C\left(P_{0}\right) T\left(P_{0}\right)=\int_{L}\left[Q\left(P, P_{0}\right) T(P)-R\left(P, P_{0}\right) q(P)\right] d L \quad \forall P_{0} \in L \cup \Omega
$$

where, $Q=\frac{1}{2 \pi r} \frac{\partial r}{\partial n}, \quad R=\frac{1}{2 \pi} \ln r . P_{0}$ and $P$ are the source and the integral points, respectively. $T$ and $q=\frac{\partial T}{\partial n}$ are temperature and outer normal temperature derivative on boundaries, respectively. For two-dimensional problems, when the source point $P_{0}$ is very close to the integral element $\Delta L$, the terms, which have same orders of singularity as $\ln r$ and $1 / \mathrm{r}$ in integral equation (1), are called nearly weak singular integral and nearly singular integrals, respectively. In the boundary element analysis of a thin body, one of the most important work is to evaluate the nearly singular integral effectively.

\subsection{Treatment of nearly singular integrals}

Using the singularity isolating technology, the nearly singular integral in equation (1) cab be given as

$$
\int_{\Delta L} \frac{1}{2 \pi r} \frac{\partial r}{\partial n} T d L=\int_{\Delta L} \frac{1}{2 \pi r} \frac{\partial r}{\partial n}\left[T(P)-T\left(P_{0}^{\prime}\right)\right] d L+T\left(P_{0}^{\prime}\right) \int_{\Delta L} \frac{1}{2 \pi r} \frac{\partial r}{\partial n} d L
$$

By use of the relation $\frac{\partial r}{\partial n}=\vec{n} \bullet \vec{r} / r=\frac{r d \theta}{d L}$ (see Fig. 1), the nearly singular term at the right hand side of the integral (2) can be integrated in a closed form

$$
\int_{\Delta L} \frac{1}{2 \pi r} \frac{\partial r}{\partial n} d L=\frac{1}{2 \pi} \int_{\Delta L} d \theta=\left.\frac{1}{2 \pi} \theta\right|_{\theta_{1}} ^{\theta_{2}}
$$

From integral (3), one can see no mater how close the source point $P_{0}$ to the integral element, a exact solution of the integral (3) can be obtained directly. In equation (2), $P_{0}^{\prime}$ is the closest point on the integral element to the source point $P_{0}$ （see Fig.1).

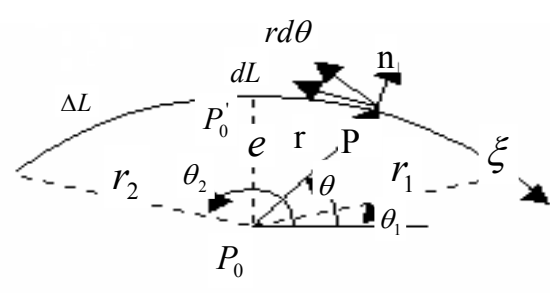

Fig.1 Local polar coordinate system of an integral element

In the natural coordinate system, the origin point 0 of the local coordinate was chosen at $P_{0}^{\prime}$ (see Fig.2). When the source point $P_{0}$ approaches the point $P_{0}^{\prime}$, using the Taylor's extension of the shape function, the principle part of the first term in the right hand side of equation (2) is $\xi / r$, and can be expressed as

$$
\int_{\Delta L} \frac{\xi}{r} d L=\int_{-1}^{1} \frac{\xi}{\sqrt{e^{2}+(\xi|J|)^{2}}}|J| d \xi=\int_{-1}^{0} \frac{\xi}{\sqrt{d^{2}+\xi^{2}}} d \xi+\int_{0}^{1} \frac{\xi}{\sqrt{d^{2}+\xi^{2}}} d \xi
$$


where $e$ is distance between source point $P_{0}$ and the integral element $\Delta L, \quad|J|$ is Jacobian, and non-dimensional distance between $P_{0}$ and the integral element $\Delta L$ is $d=e /|J|$. When $d$ is very small, the function $\xi / \sqrt{\xi^{2}+d^{2}}$ changes sharply near the origin 0 . Therefore, its numerical integral performed by the Gaussian quadrature is not efficient. A non-linear transformation is proposed in Ref. [6] to solve the problem. The nearly singular

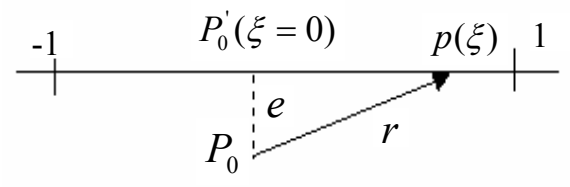

Fig.2 Integral element in natural coordinate system integral after the non-linear transformation $\xi=\eta^{m}$ can be expressed as

$$
\int_{0}^{1} \sqrt{\frac{\xi^{2}}{d^{2}+\xi^{2}}} d \xi=\int_{0}^{1} m \eta^{m-1} \sqrt{\frac{\eta^{2 m}}{d^{2}+\eta^{2 m}}} d \eta
$$

when a suitable value $m$ is chosen, the kernel function $m \eta^{m-1} \sqrt{\frac{\eta^{2 m}}{d^{2}+\eta^{2 m}}}$ changes slower, so that an accurate solution of integral (4) can be evaluated effectively by the Gaussian quadrature .

\subsection{Treatment of nearly weakly-singular integrals}

The nearly week singular integral in equation (1) can also be evaluated by the same non-linear transformation $\xi=\eta^{m}$.

$$
\int_{\Delta L} \ln r N(\xi) d L=\int_{-1}^{0} \frac{1}{2} \ln \left[\left(\xi^{2}+d^{2}\right)|J|^{2}\right] N(\xi)|J| d \xi+\int_{0}^{1} \frac{1}{2} \ln \left[\left(\xi^{2}+d^{2}\right)|J|^{2}\right] N(\xi)|J| d \xi
$$

where

$$
\int_{0}^{1} \frac{1}{2} \ln \left[\left(\xi^{2}+d^{2}\right)|J|^{2}\right] N(\xi)|J| d \xi=\int_{0}^{1} \frac{m}{2} \eta^{m-1} \ln \left[\left(\eta^{2 m}+d^{2}\right)|J|^{2}\right] N\left(\eta^{m}\right)|J| d \eta
$$

Similarly, when a suitable value $m$ is chosen, an accurate solution of the weakly nearly-singular integral (6) can be obtain effectively by the Gaussian quadrature .

\section{BEM formulation for steady-state thermal analysis of thermal barrier coated components}

For the steady-state thermal analysis of a multi-layer structure in Fig.3, the boundary integral equations for the later $\beta$ can be given from the equation (1)

$$
\begin{aligned}
& C^{(\beta)}\left(P_{0}\right) T^{(\beta)}\left(P_{0}\right)=\int_{L}\left[Q^{(\beta)}\left(P, P_{0}\right) T^{(\beta)}(P)-R^{(\beta)}\left(P, P_{0}\right) q^{(\beta)}(P)\right] d L, \quad \forall P_{0} \in L \cup \Omega \\
& \mathbf{Q}^{(i)} \mathbf{T}^{(i)}=\mathbf{R}^{(i)} \mathbf{q}^{(i)}, \quad i=1,2,3 \quad(8)
\end{aligned}
$$


where, $\mathbf{Q}^{(i)}$ and $\mathbf{R}^{(i)}$ denote coefficient matrixes of the integrated fundamental solutions $Q\left(P, P_{0}\right)$ and $R\left(P, P_{0}\right)$ multiplied by the shape function, respectively. $\mathbf{T}^{(i)}$ and $\mathbf{q}^{(i)}$ denote boundary temperature vector and its outer normal derivative vector, with superscript denoting layer number. The boundary of the region $\Omega_{j}$ of $j$ th layer can be split into two part, $L=L_{j-1} \cup L_{j}, \quad j=1,2,3$, with $L_{j-1}$ and $L_{j}$ denote the inter and outer boundaries of $\Omega_{j}$. By partitioning the temperature vector and the derivatives vector on the inter and outer

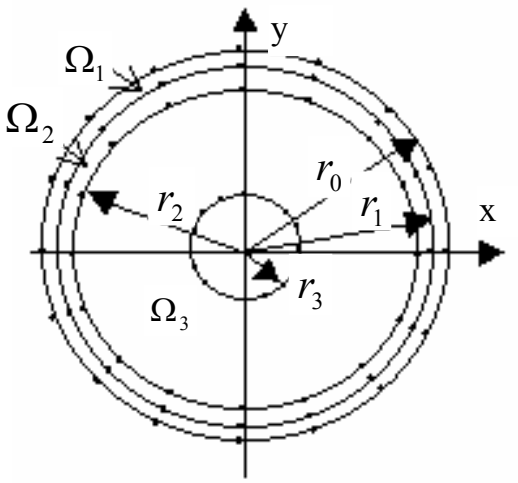

Fig. 3 Multi-layer structure boundaries equation (8) can be rewritten as

$$
\begin{array}{ll}
\Omega_{1}: & \mathbf{Q}_{0}^{(1)} \mathbf{T}_{0}^{(1)}+\mathbf{Q}_{1}^{(1)} \mathbf{T}_{1}^{(1)}=\mathbf{R}_{0}^{(1)} \mathbf{q}_{0}^{(1)}+\mathbf{R}_{1}^{(1)} \mathbf{q}_{1}^{(1)} \\
\Omega_{2}: & \mathbf{Q}_{1}^{(2)} \mathbf{T}_{1}^{(2)}+\mathbf{Q}_{2}^{(2)} \mathbf{T}_{2}^{(2)}=\mathbf{R}_{1}^{(2)} \mathbf{q}_{1}^{(2)}+\mathbf{R}_{2}^{(2)} \mathbf{q}_{2}^{(2)} \\
\Omega_{3}: & \mathbf{Q}_{2}^{(3)} \mathbf{T}_{2}^{(3)}+\mathbf{Q}_{3}^{(3)} \mathbf{T}_{3}^{(3)}=\mathbf{R}_{2}^{(3)} \mathbf{q}_{2}^{(3)}+\mathbf{R}_{3}^{(3)} \mathbf{q}_{3}^{(3)}
\end{array}
$$

where the subscript denotes the boundary number, while superscript denotes the layer number.

Let boundary temperature vector and the derivatives vector

$$
\begin{aligned}
& \mathbf{q}_{0}=\mathbf{q}_{0}^{(1)}, \quad \mathbf{q}_{1}=\mathbf{q}_{1}^{(1)}, \quad \mathbf{q}_{2}=\mathbf{q}_{2}^{(3)}, \quad \mathbf{q}_{3}=\mathbf{q}_{3}^{(3)} \\
& \mathbf{T}_{0}=\mathbf{T}_{0}^{(1)}, \quad \mathbf{T}_{1}=\mathbf{T}_{1}^{(1)}, \quad \mathbf{T}_{2}=\mathbf{T}_{2}^{(3)}, \quad \mathbf{T}_{3}=\mathbf{T}_{3}^{(3)}
\end{aligned}
$$

When the interfaces are perfectly bounded, continuity conditions on the interfaces can be given as: the temperatures on the both side of the interface are the same, while the thermal flux on the both side of the interface are equal and opposite.

$$
\lambda_{1} \mathbf{q}_{1}^{(1)}=-\lambda_{2} \mathbf{q}_{1}^{(2)}, \quad \lambda_{2} \mathbf{q}_{2}^{(2)}=-\lambda_{3} \mathbf{q}_{2}^{(3)}, \quad \mathbf{T}_{1}=\mathbf{T}_{1}^{(1)}=\mathbf{T}_{1}^{(2)}, \quad \mathbf{T}_{2}=\mathbf{T}_{2}^{(2)}=\mathbf{T}_{2}^{(3)}
$$

where $\lambda_{i}$ is the thermal conduct coefficient of material in the $i$ th layer. Suppose the temperatures on the surface $L_{0}$ (and $L_{3}$, if exists) are know, and its derivatives unknown, without lose of the generality. The temperature and its derivatives on the boundary $L_{1}$ and $L_{2}$ are unknown, but meet the continuity conditions (11). After substitution of the equations (10) and (11) into the equation (9), rewriting the equation by placing the know and the unknown boundary values and their corresponding coefficient matrixes at the right and left hand sides, respectively, boundary element linear equation system of the thermal analysis of a three-layer structure can be deduced 


$$
\left[\begin{array}{cccccc}
-\mathbf{R}_{0}^{(1)} & \mathbf{Q}_{1}^{(1)} & \mathbf{0} & \mathbf{0} & -\mathbf{R}_{1}^{(1)} & \mathbf{0} \\
\mathbf{0} & \mathbf{Q}_{1}^{(2)} & \mathbf{Q}_{2}^{(2)} & \mathbf{0} & \frac{\lambda_{1}}{\lambda_{2}} \mathbf{R}_{1}^{(2)} & \frac{\lambda_{3}}{\lambda_{2}} \mathbf{R}_{2}^{(2)} \\
\mathbf{0} & \mathbf{0} & \mathbf{Q}_{2}^{(3)} & -\mathbf{R}_{3}^{(3)} & \mathbf{0} & -\mathbf{R}_{2}^{(3)}
\end{array}\right]\left[\begin{array}{c}
\mathbf{q}_{0} \\
\mathbf{T}_{1} \\
\mathbf{T}_{2} \\
\mathbf{q}_{3} \\
\mathbf{q}_{1} \\
\mathbf{q}_{2}
\end{array}\right]=\left[\begin{array}{cc}
-\mathbf{Q}_{0}^{(1)} & \mathbf{0} \\
\mathbf{0} & \mathbf{0} \\
\mathbf{0} & -\mathbf{Q}_{3}^{(3)}
\end{array}\right]\left[\begin{array}{c}
\mathbf{T}_{0} \\
\mathbf{T}_{3}
\end{array}\right]
$$

If the third kind of boundary condition, $\lambda q=\alpha\left(T_{f}-T\right)$, is given on the outer and inner boundaries, or in general, it can also be expressed as $q+b T=c$, in which $\alpha$ denotes the boundary thermal exchange coefficient, $T_{f}$ the around medium temperature, coefficients $b=\alpha / \lambda, \quad c=\alpha T_{f} / \lambda$. Boundary conditions on surfaces $L_{0}$ and $L_{3}$ in matrix form can be expressed as: $\mathbf{q}_{0}=\mathbf{c}_{0}-\mathbf{B}_{0} \mathbf{T}_{0}, \mathbf{q}_{3}=\mathbf{c}_{3}-\mathbf{B}_{3} \mathbf{T}_{3}$, where $\mathbf{c}_{0}$ and $\mathbf{c}_{3}$ are the vectors of the coefficient $c$ on the boundaries $L_{0}$ and $L_{3}$, respectively, $\mathbf{B}_{0}$ and $\mathbf{B}_{3}$ are the diagonal matrices of coefficient $b$ on the boundaries. $\circ$ By use of the third kind of boundary condition mentioned above, the linear equation system of the boundary element method for thermal analysis of a multi-layer structure can be given as

$$
\left[\begin{array}{cccccc}
\mathbf{Q}_{0}^{(1)}-\mathbf{R}_{0}^{(1)} \mathbf{B}_{0} & \mathbf{Q}_{1}^{(1)} & \mathbf{0} & \mathbf{0} & -\mathbf{R}_{1}^{(1)} & \mathbf{0} \\
\mathbf{0} & \mathbf{Q}_{1}^{(2)} & \mathbf{Q}_{2}^{(2)} & \mathbf{0} & \frac{\lambda_{1}}{\lambda_{2}} \mathbf{R}_{1}^{(2)} & \frac{\lambda_{3}}{\lambda_{2}} \mathbf{R}_{2}^{(2)} \\
\mathbf{0} & \mathbf{0} & \mathbf{Q}_{2}^{(3)} & \mathbf{Q}_{3}^{(3)}-\mathbf{R}_{3}^{(3)} \mathbf{B}_{3} & \mathbf{0} & -\mathbf{R}_{2}^{(3)}
\end{array}\right]\left[\begin{array}{c}
\mathbf{T}_{0} \\
\mathbf{T}_{1} \\
\mathbf{T}_{2} \\
\mathbf{T}_{3} \\
\mathbf{q}_{1} \\
\mathbf{q}_{2}
\end{array}\right]=\left[\begin{array}{cc}
\mathbf{R}_{0}^{(1)} & \mathbf{0} \\
\mathbf{0} & \mathbf{0} \\
\mathbf{0} & \mathbf{R}_{3}^{(3)}
\end{array}\right]\left[\begin{array}{c}
\mathbf{c}_{0} \\
\mathbf{c}_{3}
\end{array}\right]
$$

All the unknowns on the boundaries can be solved from equations (12) or (13). Then the temperature at inner point can be obtained from equation (7), if needed.

\section{Boundary integral equations for thermal stress analysis under complex loads and treatment of nearly singular integrals}

The boundary integral equations of an isotropy elastic body under thermal and centrifugal loads can be expressed as ${ }^{[10]}$ :

$$
\begin{aligned}
& C_{i j}\left(P_{0}\right) u_{j}\left(P_{0}\right)=\int_{L}\left[U_{i j}\left(P, P_{0}\right) t_{j}(P)-T_{i j}\left(P, P_{0}\right) u_{j}(P)\right] d L(P)+ \\
& +\int_{L}\left[S_{i}\left(P, P_{0}\right) \Delta T-V_{i}\left(P, P_{0}\right) \frac{\partial T}{\partial n}\right] d L(P)+\int_{L} a_{i}\left(P, P_{0}\right) d L(P), \forall P_{0} \in L \cup \Omega
\end{aligned}
$$

where, for the plane strain problems, the fundamental solution functions can be given as

$$
\begin{aligned}
& U_{i j}\left(P, P_{0}\right)=\frac{1}{8 \pi G(1-v)}\left[-(3-4 v\} \delta_{i j} \ln r+r,{ }_{i} r,{ }_{j}\right] \\
& T_{i j}\left(P, P_{0}\right)=\frac{-1}{4 \pi(1-v) r}\left\{\frac{\partial r}{\partial n}\left[(1-2 v) \delta_{i j}+2 r,{ }_{i} r,{ }_{j}\right]+(1-2 v)\left(r,{ }_{j} n_{i}-r,{ }_{i} n_{j}\right)\right\} \\
& S_{i}=\frac{-\alpha(1+v)}{4 \pi(1-v)}\left[(\ln r+1 / 2) n_{i}+\frac{\partial r}{\partial n} r,_{i}\right], \quad V_{i}=\frac{-\alpha(1+v)}{4 \pi(1-v)}(\ln r+1 / 2) r,{ }_{i} r
\end{aligned}
$$


$a_{i}=\frac{-\rho \omega^{2} r}{8 \pi G}\left\{(2 \ln r+1 / 2)\left[\frac{\partial r}{\partial n} y_{i}-\frac{1}{2(1-v)} y_{m} r,{ }_{m} n_{i}\right]-\frac{1-2 v}{2(1-v)} r n_{i} \ln r\right\}$

in which $P_{0}$ and $P$ are the source and the integral points, respectively. $u_{i}$ and $t_{i}$ are the displacements and the traction on a boundary node $P$, respectively. $\Delta T$ and $q=\frac{\partial T}{\partial n}$ are the temperature change and the temperature derivatives on the outer normal direction of the boundary, respectively. $y_{k}$ is distance vector between integral point $\mathrm{P}$ and the rotating axis。In the integral equation (14), the body force integral has been transformed to the integral over the boundary. For the plane stress problems, the poison's ratio $v$ and thermal extension coefficient $\alpha$ in (14)-(18) will be replaced by $v /(1+v)$ and $\alpha(1+v) /(1+2 v)$, respectively.

For stress analysis of a two-dimensional thin body, similar nearly weak singular integral and nearly singular integral in Cauchy principle value exist. An effective integral scheme should be chosen to deal with this kind of the nearly singular integral problem.

\subsection{Treatment of nearly singular integrals}

By use of the singularity isolating technology, the nearly singular integral in equation (14) can be expressed as [6]:

$$
\int_{\Delta L} T_{i j}\left(P, P_{0}\right) u_{j}(P) d L=\int_{\Delta L} T_{i j}\left(P, P_{0}\right)\left[u_{j}(P)-u_{j}\left(P_{0}^{\prime}\right)\right] d L+u_{j}\left(P_{0}^{\prime}\right) \int_{\Delta L} T_{i j}\left(P, P_{0}\right) d L
$$

The last integral in (19) can be integrated in a closed form as [6]:

$$
\begin{gathered}
\int_{\Delta L} T_{i j}\left(P, P_{0}\right) d L=\left.C_{1} C_{2}\left(e_{1 i} e_{2 j}-e_{2 i} e_{1 j}\right) \ln r\right|_{r_{1}} ^{r_{2}}+C_{1}\left\{C_{2} \delta_{i j} \theta+\delta_{1 i} \delta_{1 j}\left(\theta+\frac{1}{2} \sin 2 \theta\right)\right. \\
\left.-\frac{1}{2}\left(\delta_{1 i} \delta_{2 j}+\delta_{2 i} \delta_{1 j}\right) \cos 2 \theta+\delta_{2 i} \delta_{2 j}\left(\theta-\frac{1}{2} \sin 2 \theta\right)\right\}\left.\right|_{\theta_{1}} ^{\theta_{2}}
\end{gathered}
$$

where, $C_{1}=-1 / 4 \pi(1-v), C_{2}=1-2 v$, the angles and the radius in the local polar coordinate system are shown in Figure 1. From equation (20) one can see that no matter what shape of the element is, and how close the source point $P_{0}$ to the integral element $\Delta L$, an exact solution of the integral (20) can be obtained directly. In the equation (19), $P_{0}^{\prime}$ is the closest point on the integral element to the source point $P_{0}$

(see Fig.1).

In the natural coordinate system, the origin $O$ of the local coordinate system is placed at the point $P_{0}^{\prime}$ (see Fig.2). When the source point $P$ approaches point $P_{0}^{\prime}$ on the integral element, the first integral in the right hand side of equation (19) can be written as

$$
\int_{\Delta L} \frac{f(\xi) \xi}{r} d L=\int_{-1}^{1} \frac{f(\xi) \xi}{\sqrt{e^{2}+(\xi|J|)^{2}}}|J| d \xi=\int_{-1}^{0} \frac{f(\xi) \xi}{\sqrt{d^{2}+\xi^{2}}} d \xi+\int_{0}^{1} \frac{f(\xi) \xi}{\sqrt{d^{2}+\xi^{2}}} d \xi
$$


by the discritization of the shape function $N_{\alpha}(\xi)$, where $f(\xi)=\frac{r}{\xi} T_{i j}\left[N_{\alpha}(\xi)-N_{\alpha}(0)\right]$ is a non-singular function. When the distance $e$ between the source point $P_{0}$ and the integral element $\Delta L$ gets small, the principal part of the kernel function in the integral (21) will be $\xi / \sqrt{\xi^{2}+d^{2}}$ (see Fig.2), where $d=e /|J|$ denotes the non-dimensional distance between point $P_{0}$ and the integral element $\Delta L,|J|$ the Jacobian of coordinate transformation. When $d$ is very small, but not zero, the evaluation of function $\xi / \sqrt{\xi^{2}+d^{2}}$ by Gaussian quadrature is inefficient, as the integral kernel changes sharply near the origin 0 . By virtue of non-linear transformation $\xi=\eta^{m[6]}$, equation (21) can be expressed as:

$$
\int_{0}^{1} \frac{f(\xi) \xi}{\sqrt{d^{2}+\xi^{2}}} d \xi=\int_{0}^{1} \frac{f\left(\eta^{m}\right) m \eta^{2 m-1}}{\sqrt{d^{2}+\xi^{2}}}|J| d \eta
$$

The equation (22) can be evaluated effectively by use of Gaussian quadrature, as the change rate of the kernel function gets uniform, when a suitable $m$ is used.

\subsection{Treatment of nearly weak singular integrals}

The nearly weak singular integral in the kernel $U_{i j}$ and $S_{i}$ in equation (14) can be evaluated by Gaussian quadrature, after the same non-linear transformation $\xi=\eta^{m[6]}$ is used.

$$
\int_{\Delta L} \ln r N(\xi) d L=\int_{-1}^{1} \frac{1}{2} \ln \left[\left(\xi^{2}+d^{2}\right)|J|^{2}\right] N(\xi)|J| d \xi
$$

Generally, the above integral has to be integrated over two sub-elements as equation (6). The integral over each sub-element has to be transformed to the $[0,1]$ region first to do the non-linear transformation. Then it can be evaluated by the Gaussian quadrature. In order to shorten the computing time and simplify the programming, the different nonlinear transformations $\xi=\eta^{m}$ and $\xi=-|\eta|^{m}$ can be used in one integral element at $\xi \geq 0$ and $\xi<0$ regions, respectively, without the subdivision of the integral element. The parameter $m$ can generally be chosen larger than one. The better choice of $m$ is about 3 in this paper. The non-linear transformation is not necessary to evaluate the non-singular integral kernels $a_{i}$ of centrifugal load, and $V_{i}$ of thermal load in equation (14).

\section{BEM linear equation system of thermal elastic analysis of thermal barrier coated components under complex loads}

For thermal elastic analysis of a multi-layer structure under centrifugal and thermal loads as in Fig.2, boundary equation integral can be written, from equation (14), as

$$
\begin{array}{r}
C_{i j}^{(\beta)}{ }_{i j}\left(P_{0}\right) u_{j}^{(\beta)}\left(P_{0}\right)=\int_{L}\left[U_{i j}^{(\beta)}\left(P, P_{0}\right) t_{j}^{(\beta)}(P)-T_{i j}^{(\beta)}\left(P, P_{0}\right) u_{j}^{(\beta)}(P)\right] d L+\int_{L} A_{i}^{(\beta)}, \\
\forall P_{0} \in L \cup \Omega
\end{array}
$$


where $L$ denotes the boundaries of the layer, superscript $\beta$ denotes layer number. The last integral in equation (24) is from the boundary integral of the thermal and / or the centrifugal loads. For a three-layer structure, the matrix form of the linear equation system of the $i$ th layer $\Omega_{i}$ can be obtained from equation (24), after discritization

$$
\mathbf{T}^{(i)} \mathbf{u}^{(i)}=\mathbf{U}^{(i)} \mathbf{t}^{(i)}+A^{(i)}, \quad i=1,2,3
$$

where, $\mathbf{T}^{(i)}$ and $\mathbf{U}^{(i)}$ denote the coefficient matrices formed by the integration of the fundamental solution functions $T_{i j}\left(P, P_{0}\right)$ and $U_{i j}\left(P, P_{0}\right)$ multiplied by the shape function, respectively. A denotes a load vector due to a temperature change and / or centrifugal force. $\mathbf{u}^{(i)}$ and $\mathbf{t}^{(i)}$ denote the boundary displacement and traction vectors of every region. Superscript $i$ denotes layer number. Suppose the boundary of the $j$ th region $\Omega_{j}$ be $L=L_{j-1} \cup L_{j}, \quad j=1,2,3$, where $L_{j-1}$ and $L_{j}$ denote the inner and outer boundaries of the region $\Omega_{j}$, respectively. After dividing the displacement vector and traction vector into two parts, the ones on inner boundary and on outer boundary, equation (25) can be rewritten as

$$
\begin{array}{ll}
\Omega_{1}: & \mathbf{T}_{0}^{(1)} \mathbf{u}_{0}^{(1)}+\mathbf{T}_{1}^{(1)} \mathbf{u}_{1}^{(1)}=\mathbf{U}_{0}^{(1)} \mathbf{t}_{0}^{(1)}+\mathbf{U}_{1}^{(1)} \mathbf{t}_{1}^{(1)}+\mathbf{A}^{(1)} \\
\Omega_{2}: & \mathbf{T}_{1}^{(2)} \mathbf{u}_{1}^{(2)}+\mathbf{T}_{2}^{(2)} \mathbf{u}_{2}^{(2)}=\mathbf{U}_{1}^{(2)} \mathbf{t}_{1}^{(2)}+\mathbf{U}_{2}^{(2)} \mathbf{t}_{2}^{(2)}+\mathbf{A}^{(2)} \\
\Omega_{3}: & \mathbf{T}_{2}^{(3)} \mathbf{u}_{2}^{(3)}+\mathbf{T}_{3}^{(3)} \mathbf{u}_{3}^{(3)}=\mathbf{U}_{2}^{(3)} \mathbf{t}_{2}^{(3)}+\mathbf{U}_{3}^{(3)} \mathbf{t}_{3}^{(3)}+\mathbf{A}^{(3)}
\end{array}
$$

where the subscript denotes the boundary number, the superscript denotes the layer or region number. For the sake of convenience, let boundary value vectors be some new simple vectors.

$$
\begin{aligned}
& \mathbf{t}_{0}=\mathbf{t}_{0}^{(1)}, \quad \mathbf{t}_{1}=\mathbf{t}_{1}^{(1)}, \quad \mathbf{t}_{2}=\mathbf{t}_{2}^{(3)}, \quad \mathbf{t}_{3}=\mathbf{t}_{3}^{(3)} \\
& \mathbf{u}_{0}=\mathbf{u}_{0}^{(1)}, \quad \mathbf{u}_{1}=\mathbf{u}_{1}^{(1)}, \quad \mathbf{u}_{2}=\mathbf{u}_{2}^{(3)}, \quad \mathbf{u}_{3}=\mathbf{u}_{3}^{(3)}
\end{aligned}
$$

If the interfaces are perfectly bounded, the continued conditions of the displacements and traction on the interfaces can be given as

$$
-\mathbf{t}_{1}=\mathbf{t}_{1}^{(2)}, \quad-\mathbf{t}_{2}=\mathbf{t}_{2}^{(2)}, \quad \mathbf{u}_{1}=\mathbf{u}_{1}^{(2)}, \quad \mathbf{u}_{2}=\mathbf{u}_{2}^{(2)}
$$

Suppose boundary conditions are well posted on the outer boundary and the inner boundary if exist, while displacements and traction on interfaces $L_{1}$ and $L_{2}$ are unknown, but fulfil the continuity condition (28). Suppose the traction is known, but displacements, except for the ones to constrain the rigid body movement, are unknown on the outer and inner boundaries, without lose of generalization. The matrix form of the linear equation system for the boundary element thermal elastic analysis of a three-layer coated structure can be given as equation (29), after rearranging the known and the unknown vectors and the corresponding coefficient matrices at the right and the left hand side of equation (29), respectively. 


$$
\left[\begin{array}{cccccc}
\mathbf{T}_{0}^{(1)} & \mathbf{T}_{1}^{(1)} & \mathbf{0} & \mathbf{0} & -\mathbf{U}_{1}^{(1)} & \mathbf{0} \\
\mathbf{0} & \mathbf{T}_{1}^{(2)} & \mathbf{T}_{2}^{(2)} & \mathbf{0} & \mathbf{U}_{1}^{(2)} & \mathbf{U}_{2}^{(2)} \\
\mathbf{0} & \mathbf{0} & \mathbf{T}_{2}^{(3)} & \mathbf{T}_{3}^{(3)} & \mathbf{0} & -\mathbf{U}_{2}^{(3)}
\end{array}\right]\left[\begin{array}{c}
\mathbf{u}_{0} \\
\mathbf{u}_{1} \\
\mathbf{u}_{2} \\
\mathbf{u}_{3} \\
\mathbf{t}_{1} \\
\mathbf{t}_{2}
\end{array}\right]=\left[\begin{array}{cc}
\mathbf{U}_{0}^{(1)} & \mathbf{0} \\
\mathbf{0} & \mathbf{0} \\
\mathbf{0} & \mathbf{U}_{3}^{(3)}
\end{array}\right]\left[\begin{array}{l}
\mathbf{t}_{0} \\
\mathbf{t}_{3}
\end{array}\right]+\left[\begin{array}{l}
\mathbf{A}^{(1)} \\
\mathbf{A}^{(2)} \\
\mathbf{A}^{(3)}
\end{array}\right]
$$

Let $N_{0} 、 N_{1} 、 N_{2} 、 N_{3}$ and $M_{0} 、 M_{1} 、 M_{2} 、 M_{3}$ denote the total numbers of nodes and elements on the every boundaries, respectively. The total number of unknown values in the boundary element equation system (29) is $N F=\left(2 N_{0}+4 N_{1}+4 N_{2}+2 N_{3}\right)$, which is the length of the unknown vector in the left-hand side of the equation (29). All the unknown displacements and traction on the boundaries can be solved from equation (29). Furthermore, the other stress components on the boundaries and the displacements and stresses in the region can be obtained from equation (14) and a related formulation, if necessary.

\section{Numerical examples}

\section{Thermal analyses of a thermal barrier coated component}

A two-dimensional section model of a hollow long shaft coated by a bond layer and a ceramic layer shown in Fig. 3 was chosen. The outer radius $r_{0}$ of the ceramic layer is $0.1 \mathrm{~m}$, and the inner radius $r_{3}$ of the shaft is $0.005 \mathrm{~m}$. The thickness of the two outer layers $h$ varied from $0.01 \mathrm{~m}$ to $10^{-10} \mathrm{~m} .8$ quadratic boundary elements were used on the each boundary of the layers. 32 boundary elements and 64 nodes were used for the whole model. Two groups of material parameters were used for the following two numerical examples.

Example 1: Same thermal conduct parameter $\lambda_{i}=25 \mathrm{~W} /\left(\mathrm{m} \bullet{ }^{0} \mathrm{C}\right), \mathrm{i}=1,2,3$ was used for the three-layer model. The thermal exchange coefficients $h_{i}$ and air temperatures $T_{f i}$ on the inner and outer boundaries are $h_{0}=8000 \mathrm{~W} /\left(\mathrm{m} \bullet{ }^{0} \mathrm{C}\right), h_{3}=6000 \mathrm{~W} /\left(\mathrm{m} \bullet{ }^{0} \mathrm{C}\right)$, $T_{f 0}=1005.2158^{\circ} \mathrm{C}, T_{f 3}=360.9132^{\circ} \mathrm{C}$. The theoretic solution of the temperature distribution $T$ along radial direction can be deduced easily, which is the same as one of a single-material cylinder of the same size. $T=500+500 * \ln \left(r / r_{3}\right) / \ln \left(r_{0} / r_{3}\right)^{0} C$. Its derivatives along radial direction can be obtained easily from the above temperature solution. Non-dimensional temperature $\left(T_{1} / T_{0}\right)$ and its normal derivatives $\left[\left(d T_{1} / d r\right) * r_{0} / T_{0}\right]$ at interface $L_{1}\left(r=r_{1}\right)$ versus non-dimensional coating thickness $n=\log \left(r_{0} / h\right)$ were given in Fig. 4 .

Example 2: Three thermal conduct coefficients $\lambda_{i}$ for the material parameters in the three layers are $\lambda_{1}=1 \mathrm{~W} /(\mathrm{m} \bullet \mathrm{k}), \lambda_{2}=\lambda_{3}=25 \mathrm{~W} /(\mathrm{m} \bullet \mathrm{k})$. The boundary conditions for the inner and the outer boundaries are the first kind. The temperatures on outer and inner 
boundaries are $T_{0}=1000^{\circ} \mathrm{C}, T_{3}=500^{\circ} \mathrm{C}$, respectively. Non-dimensional temperature $\left(T_{1} / T_{0}\right)$ and its derivatives $\left[\left(d T_{1} / d r\right) * r_{0} / T_{0}\right]$ on interface $L_{1}\left(r=r_{1}\right)$ versus the non-dimensional coating thickness $n=\log \left(r_{0} / h\right)$ were given in Fig.5 .The temperature distribution in region $\Omega_{1}$ can be given as $T=T_{0}-Q \ln \left(r_{0} / r_{1}\right) /\left(2 \pi \lambda_{1}\right)^{[11]}$, where radial thermal flux $Q=2 \pi\left(T_{0}-T_{3}\right) /\left(\ln \left(r_{0} / r_{1}\right) / \lambda_{1}+\ln \left(r_{1} / r_{2}\right) / \lambda_{2}+\ln \left(r_{2} / r_{3}\right) / \lambda_{3}\right)$.

Thermal stress analyses of a thermal barrier coated component

The same model with same size in Fig.3 was used. The material parameters are shown in Table 1. Three types of loads were applied in the following three examples.

Example 3: a uniform traction, $\mathrm{p}=100 \mathrm{MPa}$, along radial direction were applied on the outer boundary of the ceramic layer, while the inner boundary was free.

Example 4: Temperatures on the outer and the inner boundary were $T_{0}=1000{ }^{\circ} \mathrm{C}$, $T_{3}=500{ }^{\circ} \mathrm{C}$. The theoretic solution of temperature was given in Example $2^{[11]}$. But the numerical solutions of the temperature and its derivatives were obtained by boundary element thermal analysis in this paper.

Example 5: The shaft was rotating with a speed $n=30000$ revolutions per minute. The same element model in the thermal analysis in Fig. 3 was used, in which 8 elements were uniformly placed on the each boundary in the Example 3 5. A non-uniform-element model was also used in Example 5. On each circular boundary of the non-uniform model, the length of one element was token 1.2 times as long as the corresponding one in the uniform model, and the length of the next element to it was 0.8 times as long as the corresponding one in the uniform model, while the other element length was kept as one in the uniform model. All the thermal elastic analyses were in plane strain model. The shear stress on the interfaces was zero, as all the examples are axial-symmetric. The tangent tension stress and normal tension stress on interface $L_{1}$ versus the coating thickness were given in Fig. $6 \sim$ Fig. 8 .

Table 1 Material parameters

\begin{tabular}{|l|l|l|l|c|c|}
\hline Zone & $\begin{array}{l}\text { Elastic } \\
\text { module } \\
\text { E[MPa }]\end{array}$ & $\begin{array}{l}\text { Poison's } \\
\text { ratio } v\end{array}$ & $\begin{array}{l}\text { Thermal } \\
\text { extension } \\
\text { coefficient } \\
\alpha_{i}\left[{ }^{0} \mathrm{C}^{-1}\right]\end{array}$ & $\begin{array}{l}\text { Density } \rho \\
{\left[\mathrm{kg} / \mathrm{m}^{3}\right]}\end{array}$ & $\begin{array}{l}\text { Thermal conduct } \\
\text { coefficient } \lambda_{i} \\
{\left[\mathrm{~W} /\left(\mathrm{m} \bullet{ }^{0} \mathrm{C}\right)\right]}\end{array}$ \\
\hline$\Omega_{1}$ & $1 \times 10^{4}$ & 0.25 & $1 \times 10^{-5}$ & $4 \times 10^{3}$ & 1 \\
\hline$\Omega_{2}$ & $13.7 \times 10^{4}$ & 0.27 & $1.51 \times 10^{-5}$ & $4 \times 10^{3}$ & 25 \\
\hline$\Omega_{3}$ & $21 \times 10^{4}$ & 0.30 & $2 \times 10^{-5}$ & $7.98 \times 10^{3}$ & 25 \\
\hline
\end{tabular}

The symbols CBEM、Exact and Present in Fig. $4 \sim$ Fig. 8 mean the solutions by the conventional BEM, the exact solution ${ }^{[1]}$ and the present method, respectively. 


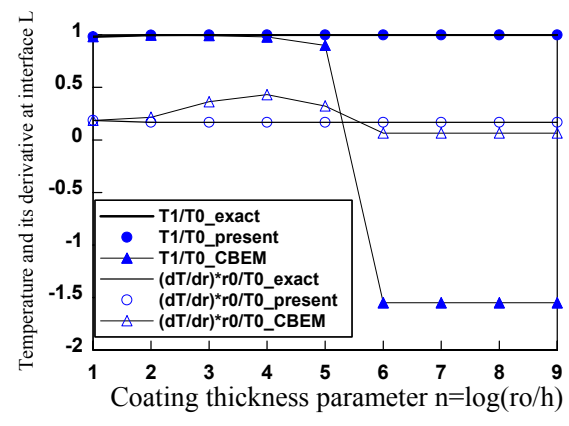

Fig.4 Temperature and its normal derivative (same materials in three layers)

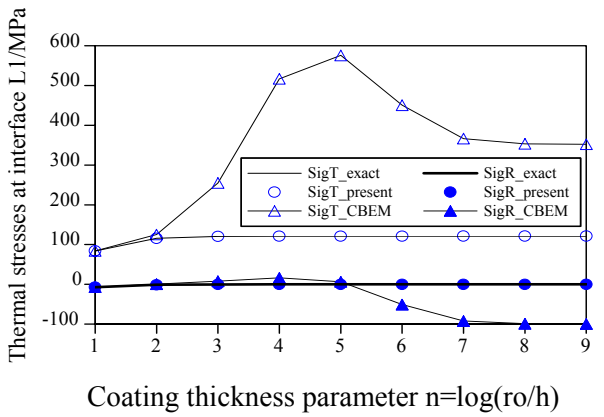

Fig.6 Interfacial thermal stresses

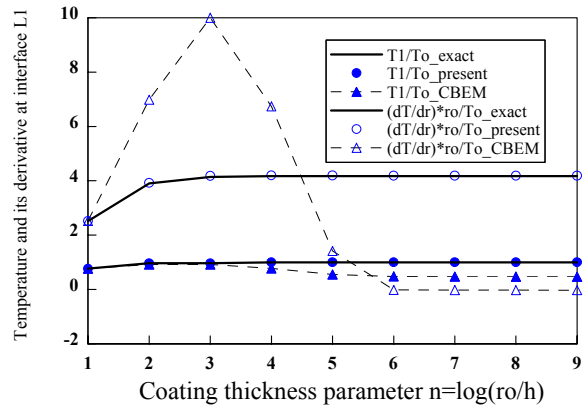

Fig.5 Temperature and its normal derivative (different materials in three layers)

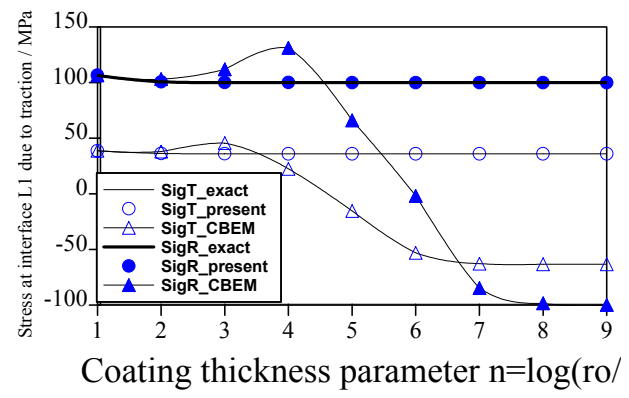

Fig.7 Interfacial stresses under traction

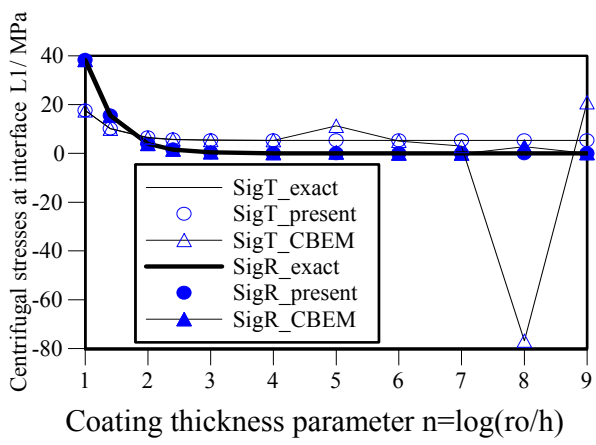

Fig.8 Interfacial centrifugal stresses (non-uniform elements) 


\section{Conclusion and discussion}

In the numerical studies reported in this paper, $10 \sim 16$ point Gaussian quadrature was used for the thermal analysis, $14 \sim 20$ point Gaussian quadrature for the stress analysis, with the power of the non-linear transformation being $3 \sim 5$ in order to obtain numerical results with enough accuracy. From the numerical results obtained, some conclusions can be made.

1) For the thermal analyses, The relative error of the normal derivatives of the temperature was one order greater than that of the temperature. The relative errors of the temperature and its normal derivatives at the interface $L_{1}$ obtained by present method was less than $1 \%$, when thickness parameter $\log \left(r_{0} / h\right)$ varied from 2 to 9 . Generally, the relative error was less than $0.2 \%$, when $\log \left(r_{0} / h\right)$ was greater than 4 . The maximum error appeared at $\log \left(r_{0} / h\right)=2 \sim 3$, as the nearly singular integrals tend to the singular integral, when $\log \left(r_{0} / h\right)$ was greater than 4 , which can also be evaluated efficiently by the present method. But if the conventional BEM was used, the larger the $\log \left(r_{0} / h\right)$ term, the larger the computational error. The maximum relative error obtained in this study was very large, greater than $620 \%$.

2) The power of the non-linear transformation, $m$, was not limited to integers, but it must be real. For thermal analysis, the maximum error of the derivatives was $5 \%$, when $m$ is limited to positive integers, while the maximum error was only $1 \%$, when a positive real parameter $m$ was used.

3) Because the thermal conductive coefficient of the ceramic was 25 times smaller than that of the substrate, the normal derivatives of the temperature at the interface $L_{1}$ in Example 2 (see Fig.5) was about 25 times greater than that in Example 1 (see Fig.4).

4) For the stress analyses, the maximum relative errors of the tangent tension stresses at interface $L_{1}$ were $0.08 \%, 0.13 \%$ and $0.23 \%$ for the thermal, traction and centrifugal loads, respectively, using the present method, in the range of $1 \leq \log \left(r_{0} / h\right) \leq 9$. While the maximum relative error of the normal tension stresses at the interface $L_{1}$ under traction load was $0.14 \%$. As the normal tension stresses at the interface $L_{1}$ under the thermal and the centrifugal loads are nearly zero at the large portion of the thickness parameter range, absolute errors, which were 0.8 and $0.06 \mathrm{MPa}$ for those two load cases, were used. Using the conventional BEM, good results can only be obtained when the thickness parameter $\log \left(r_{0} / h\right)$ was less than 2 (see Fig.6 and Fig.7). While the maximum relative errors become very large, say $480 \%, 250 \%$ and $900 \%$ for the tangent stresses under those three load cases, respectively, when the thickness parameter $\log \left(r_{0} / h\right)$ was larger than 2 .

5) For the centrifugal stresses, the maximum relative errors were $2 \sim 4.6 \%$, when the conventional BEM with uniform element size was used, with the thickness parameter 
being $2 \leq \log \left(r_{0} / h\right) \leq 4$. The errors were less than $1 \%$, with the thickness parameter being other than that range (not given in the figures). Using the conventional BEM with non-uniform element size, the maximum relative error was $5.5 \%$ and $10.4 \%$, with the thickness parameter being $1.4 \leq \log \left(r_{0} / h\right) \leq 2$ and $2.4 \leq \log \left(r_{0} / h\right) \leq 3$, respectively. And the maximum relative error became very large, approaching $900 \%$, when the thickness parameter was larger than 7. Using the present BEM with non-uniform element size, the maximum relative error was only about $1.0 \%$, with the thickness parameter being $\log \left(r_{0} / h\right) \geq 2$.

6) The temperatures, displacements and stresses at inner point near the boundary can also be calculated by the scheme of evaluating the nearly singular integral given in this paper.

7) The BEM developed in this study for the thermal and interfacial stress analyses of thermal barrier coated components are effective, which can be an useful tool for the coating design and peeling-off failure analysis of thermal barrier coated components.

\section{References}

1. Collected papers of thermal barrier coating technology in aeroengine, Chinese aviation industry 606 institute, 1999

2. Chang G C, Phucharoen W, Miller R E. Finite element thermal stress solutions for thermal barrier coatings. Surface and Coating Technology, 32: 307-325, 1987.

3. Mill T C, Chona R. FEA of thermally loaded interface crack in a ceramic coating. Engineering Fracture Mechanics, 59(2):203-214, 1998.

4. Chen B F, Hwang J, Chen I F, et al. Tensile-film-cracking model for evaluating interfacial shear strength of elastic film on ductile substrate. Surface and Coatings Technology, 126: 91-95, 2000.

5. Luo J F, Liu Y J, Berger E. Interface stress analysis for multi-coating systems using an advanced BEM. Computational Mechanics, 24: 448-455, 2000.

6. Luo J F, Liu Y J, Berger E. Analysis of two-dimensional thin-structures (from micro- to nano-scales) using the boundary element method. Computational Mechanics, 22: 404-412, 1998.

7. Chen X L, Liu Y J. Thermal stress analysis of multi-layer thin films and coatings by an advanced boundary element method. Computer Modeling in Engineering \& Science, 2(3): 337-350, 2001.

8. Nesbitt $\mathrm{J}$ A. Thermal modeling of various $\mathrm{TBC}$ in a high heat flux rocket engine. Surface and Coating Technology, 130: 141-151, 2000.

9. Fairweather G, Rizzo F J, et al.. On the numerical solution of 2D potential problems by an improved BIEM. J of Computational Physics, 31(1):96-112, 1977.

10. Brebbia C A. Progress in Boundary Element Method, Vol.2. New York: SpringerVerlag, 1984.

11. Dong Manhong. BEM for thermal and thermal stress analyses of thermal barrier coated components and its applications (in Chinese). Thesis, Northwestern Polytechnical University, Xi'an, P.R. China, 2001. 